\title{
Acute heart failure due to COVID-19 related myocardial injury and de novo hypertensive cardiomyopathy: a challenging multi-modality diagnosis
}

\author{
Matteo Pernigo ${ }^{1}$, Marco Triggiani ${ }^{1}$, Emanuele Gavazzi ${ }^{2}$, Ilaria Papa ${ }^{1}$, Alberto Vaccari ${ }^{1}$, \\ Simona Fisogni ${ }^{2}$, Ester Costantino ${ }^{1}$, and Gian Franco Pasini ${ }^{1}$ \\ ${ }^{1}$ ASST del Garda \\ ${ }^{2}$ University of Brescia
}

November 16, 2020

\begin{abstract}
We report a COVID19 case with acute heart and kidney failure in a healthy young male. Echocardiography showed severe systolic and diastolic left ventricle dysfunction, with diffuse myocardial thickening. Cardiac MRI showed aspects of focal myocarditis, and hypertensive cardiomyopathy. Renal biopsy demonstrated limited acute tubular injury, and hypertensive kidney disease. Coronary angiography excluded critical stenoses. Unlike what we initially suspected, myocardial inflammation had a limited extent in our patient; severe hypertension causing cardiomyopathy and multi-organ damage, not diagnosed before, was primarily responsible for severe illness. Correct diagnosis and guidelines-directed treatment allowed a favorable course.
\end{abstract}

Acute heart failure due to COVID-19 related myocardial injury and de novo hypertensive cardiomyopathy: a challenging multi-modality diagnosis

Authors: Matteo Pernigo ${ }^{a}$, Marco Triggiani ${ }^{\mathrm{a}}$, Emanuele Gavazzi ${ }^{\mathrm{b}}$, Ilaria Papa ${ }^{\mathrm{a}}$, Alberto Vaccari ${ }^{\mathrm{a}}$, Simona Fisogni ${ }^{c}$, Ester Costantino ${ }^{d}$, Gian Franco Pasini a

Affiliations: a Cardiology Unit, 'La Memoria' hospital, Gavardo (Brescia), Italy.

b Institute of Radiology, Department of Medical and surgical Specialities, University of Brescia, Brescia, Italy

${ }^{\mathrm{c}}$ Institute of Anatomical Pathology, Department of Medical and Surgical Specialities, University of Brescia, Brescia, Italy

dNephrology Unit, 'La Memoria' hospital, Gavardo (Brescia), Italy.

Funding: none.

Conflicts of interest: none

Corresponding author: Matteo Pernigo, MD. Cardiology Unit. 'La Memoria' hospital, via Andrea Gosa 74, Gavardo (Brescia), Italy.Matteo.pernigo@gmail.com

Word count : 1548 .

Number of tables: 1

Number of figures: 4

Supplementary files, number of figures: 2 


\begin{abstract}
We report a COVID19 case with acute heart and kidney failure in a healthy young male. Echocardiography showed severe systolic and diastolic left ventricle dysfunction, with diffuse myocardial thickening. Cardiac MRI showed aspects of focal myocarditis, and hypertensive cardiomyopathy. Renal biopsy demonstrated limited acute tubular injury, and hypertensive kidney disease. Coronary angiography excluded critical stenoses. Unlike what we initially suspected, myocardial inflammation had a limited extent in our patient; severe hypertension causing cardiomyopathy and multi-organ damage, not diagnosed before, was primarily responsible for severe illness. Correct diagnosis and guidelines-directed treatment allowed a favorable course.
\end{abstract}

\title{
BACKGROUND
}

Coronavirus disease 2019 (COVID-19) is at the moment an international public health emergency, caused by a novel enveloped RNA beta-coronavirus, named 'severe acute respiratory syndrome coronavirus-2' (SARSCoV-2). The infection is mainly characterized by respiratory tract symptoms, presenting as mild flu-like illness to potentially lethal acute respiratory distress syndrome or fulminant pneumonia, but relevant cardiovascular complications and multi-organ failure can occur in severe cases [1]. Patients with known cardiovascular disease and risk factors appear more vulnerable to the infection, and hypertension is highly prevalent in patients who develop severe illness. Pathological mechanism underlying this association in still not completely elucidated: binding of the virus to angiotensin-converting enzyme 2 receptor (ACE-2), highly expressed on endothelial surfaces, with consequent renin-angiotensin-aldosterone system deregulation, and cytokine storm seem to have a relevant role [2].

We report a case of acute HF and documented cardiac inflammation during COVID-19. Multi-modality cardiac imaging and a challenging interdisciplinary medical approach lead to a de-novo diagnosis of hypertensive multi-organ damage, exacerbated by concomitant viral infection.

\section{CASE PRESENTATION}

A 45-years old man presented to our emergency department in April 2020 for atypical chest pain, fever and worsening effort dyspnoea from 7 days before. His past medical history was unremarkable and he denied alcohol and/or drugs abuse. On his arrival high blood pressure $(\mathrm{BP}=180 / 110 \mathrm{mmHg})$, bi-basal pulmonary rales and mild leg swelling were detected; oxigen saturation was $93 \%$ while breathing ambient air. Electrocardiography showed sinus tachycardia with left axis deviation, slight diffuse ST depression more prominent in inferior-lateral leads (Fig. 1A ). Blood tests revealed a significant increase in Troponin T (82 $\mathrm{ng} / \mathrm{L}$; being $14 \mathrm{ng} / \mathrm{L}$ the upper reference limit of normal - URL), serum creatinine $(3.1 \mathrm{mg} / \mathrm{dl}$; eGFR 20 $\left.\mathrm{ml} / \mathrm{min} \mathrm{ml} / \mathrm{min} / 1.73 \mathrm{~m}^{2}\right)$ and C-reactive protein $(30 \mathrm{mg} / \mathrm{L}$; being $5 \mathrm{mg} / \mathrm{L}$ the URL). At blood cell count, neutrophils were slightly augmented and lymphocytes slightly diminished. Nasopharyngeal swab was positive for SARS-CoV-2 infection. Chest X-rays and High resolution computed tomography scan of the lungs showed ground glass-like hyper density opacities limited to the peripheral portions of inferior lobes bilaterally, and a small area of consolidation in the left superior lobe (Figure 1B, 1C ). Transthoracic echocardiography was also performed and showed mild increase in $\mathrm{LV}$ dimension (end diastolic diameter: $57 \mathrm{~mm}$; end diastolic volume: $141 \mathrm{ml}, 83 \mathrm{ml} / \mathrm{m} 2 \mathrm{BSA}$ ) and moderate increase in walls thickness (interventricular septum = 13.5 $\mathrm{mm}$; posterior wall $=11 \mathrm{~mm}$ ), with a sparkling appearance. LV ejection fraction (LVEF) was severely reduced because of a diffuse parietal hypokinesis ( $\mathrm{LVEF}=30 \%$; quantified by Simpson's method). Left atrium was moderately enlarged (area $=29 \mathrm{~cm}^{2}$ ), and the mitral diastolic filling pattern was restrictive. There was a mild mitral and tricuspid regurgitation and a mild pericardial effusion was noted behind the inferior-lateral LV wall.

A few hours after admission to our cardiac intensive care unit, patient's condition degenerated abruptly; he presented $\mathrm{BP}$ elevation to $210 / 130 \mathrm{mmHg}$ and respiratory failure, requiring nitroglycerin infusion, high doses of intravenous furosemide and non-invasive ventilatory support with continuous positive airway pressure (CPAP) of $10 \mathrm{~cm} \mathrm{H20.} \mathrm{From} \mathrm{the} \mathrm{next} \mathrm{days,} \mathrm{clinical} \mathrm{conditions} \mathrm{began} \mathrm{to} \mathrm{improve,} \mathrm{allowing} \mathrm{removal} \mathrm{of} \mathrm{CPAP}$ on day 3. Therapy with bisoprolol was initiated and gradually titrated up to $3.75 \mathrm{mg} / \mathrm{die}$; amlodipine 10 $\mathrm{mg} /$ die and Alfuzosine $2 \mathrm{mg}$ bid were also introduced for BP control. For viral infection, idroxichloroquine 
(200 mg bid) and darunavir/ritonavir (800/100 mg od) were administered for 10 and 5 days respectively according to local protocol. Feverish episodes (temperature $>38^{\circ} \mathrm{C}$ ) persisted for the first 4 days. Clinical laboratory tests trend during hospitalization is summarized in Table 1 . Notably, serum creatinine remained constantly elevated; there was modest proteinuria $(228 \mathrm{mg} / \mathrm{L})$, without Bence-Jones proteinuria; serum and urine protein immunofixation excluded monoclonal free light chains.

When patient's clinical conditions were stable, echocardiogram was repeated and confirmed diffuse parietal hypokinesis with improvement in LV systolic and diastolic function (LVEF $=38 \%$ and 'pseudonormal' diastolic filling pattern) (Figure 2) . Diffuse myocardial thickening was still evident. 2D Speckle-tracking showed diffuse reduction of global longitudinal strain, more severe in anterior segments, and partial apical sparing. Cardiac magnetic resonance imaging (MRI) was performed, showing increased symmetric and circumferential wall thickness, with diffuse LV hypokinesis (LVEF 35\%) and mild hypokinesis of right ventricle (RVEF 41\%). Short tau inversion recovery and T2-Mapping sequences demonstrated the presence of focal myocardial edema, in medium and apical segments of anterior wall and anterior septum. Phase-sensitive inversion recovery sequences showed a diffuse and intramural late gadolinium enhancement (LGE) of LV myocardium in all segments, with circumferential distribution. Pericardial effusion was not present (Figure 3 ). This pattern resulted appeared consistent with hypertensive cardiomyopathy, and myocarditis of limited extent. The day after, the patient underwent cranial computed tomography, showing multiple ischemic lacunar foci of the basal ganglia bilaterally, a stabilized ischemic lesion of the right corona radiata and white matter hypodensity (Supplementary files, figure 1) . Doppler ultrasound excluded clinically relevant carotid stenosis, and significant renal arteries pathology. Renal biopsy demonstrated diffuse acute tubular injury and modest chronic interstitial inflammation, with minimal tubulitis. Severe arteriolar hyalinosis (also in preglomerular arterioles), glomerular capsular thickening and capillary basement membrane wrinkling and duplication were also present, as for chronic hypertensive kidney disease (figure 4 ). Amyloid deposits were excluded. Fifteen days after admission, the patient was discharged. One month later, after clinical and biochemical resolution of COVID-19 as demonstrated by two consecutive negative nasopharyngeal swabs, he was readmitted in our Unit for coronary angiography control. This showed diffuse atherosclerotic disease of epicardial vessels without critical stenoses (Supplementary files, Figure 2 ). A new echocardiogram demonstrated further reduction of LV volumes with improvement in EF (to 46\%). Renal function was persistently impaired (serum creatinine: $2.9 \mathrm{mg} / \mathrm{dl}$ ). Cardiac MRI performed 2 month later, showed a reduction in biventricular volumes with improvement in LVEF up to 50\%; LV parietal hypertrophy was unchanged (septal thickness: $13 \mathrm{~mm}$ ) with mild, diffuse intramural LGE; T1 and T2 sequences showed resolution of edema.

\section{DISCUSSION}

The present report describes a case of COVID 19 with acute respiratory failure, acute myocardial and kidney injury, in a patient firstly diagnosed with severe hypertension causing dilated cardiomyopathy and multiple target-organ damage. Definite diagnosis was possible through myocardial and renal tissue characterization. Notably, they were both obtained in a living patient with severe COVID 19, the first through cardiac magnetic resonance, the latter by means of kidney biopsy.

Preexisting CV disease were found to be independent predictor of in-hospital mortality in COVID-19 patients $[1,2]$. Moreover, severe illness may be complicated by acute myocardial injury, clinically expressed by cardiac troponin release, electrocardiographic abnormalities and myocardial dysfunction [3]. Cardiac involvement may occur due to ischemic (acute myocardial ischemia/infarction) or non-ischemic processes, including stressinduced cardiomyopathy and myocarditis.

Several cases of acute heart failure or cardiogenic shock caused with echo-documented and MRI-proven myocardial inflammation due to COVID-19 have been published so far $[4,5,6]$; cardiac MRI showed diffuse, extensive myocardial edema conditioning global cardiac function impairment. In our case, echocardiography during hospitalization showed diffuse myocardial thickening, with an echo-bright appearance, severe diastolic dysfunction with initially restrictive filling pattern, bi-atrial enlargement and mild pericardial effusion. Moreover, global longitudinal strain with partial apical sparing was detected at 2D-speckle tracking. These 
aspects aroused at first the suspect of a possible infiltrative cardiomyopathy, as amyloidosis. Other diagnostic hypothesis were: extensive COVID-19 related myocardial inflammation with diffuse interstitial infiltrates, or misdiagnosed hypertensive cardiomyopathy. Systemic amyloidosis was also at first suggested by the presence of kidney failure, but this hypothesis was later rejected on the basis of serum and urine immunofixation. Cardiac MRI was crucial in the diagnostic process; it confirmed diffuse myocardial thickening and systolic impairment; myocardial edema depicted by $\mathrm{T} 1$ and $\mathrm{T} 2$ sequences had a limited extent, thus suggesting that the severe cardiac impairment was not exclusively caused by myocarditis. LGE pattern appeared non suggestive for amyloidosis and supported the diagnosis of hypertensive cardiomyopathy. Interestingly, cardiac function gradually improved during and after hospitalization, in parallel with COVID-19 resolution and effective treatment of hypertension.

Kidney impairment is frequent in COVID-19 patients: more than $40 \%$ of them have proteinuria at hospital admission [7], while some critically ill patients may develop during hospitalization an acute kidney injury (AKI) [8]. The most common pathogenic factors appear related to direct renal injury (promoted by the binding of SARS-CoV-2 to ACE-2 on tubular cells and podocytes), to microthrombi formation due to hypercoagulability, and to the effects of acute systemic inflammatory response[8]. Kidney biopsy findings in our patient revealed diffuse acute tubular damage with modest interstitial inflammation, as for AKI, together with glomerular and vascular alterations consistent with chronic hypertensive nephropathy. Resolution of infective process however did not lead to a significant improvement of kidney function, thus we can speculate that in this patient the long-time undiagnosed severe hypertension was the main contributor to his kidney impairment. Cranial CT findings, suggestive for hypertensive encephalopathy, were also in keeping with a severe uncontrolled hypertension not diagnosed before.

In conclusion, our report depicts how a condition of severe, untreated hypertension can increase cardiac vulnerability to COVID-19 infection: acute inflammatory alterations, even of limited extent, may critically destabilize a preexisting organ damage of hypertensive etiology, leading to a condition of severe HF. This report highlights that in these patients the same mechanistic approach to cardiac and systemic abnormalities should be used, increasing utilization of multi-modality imaging and diagnostic tools that may help us to make a correct diagnosis.

Table 1

Laboratory tests during hospitalization

\begin{tabular}{lll}
\hline Measure & Reference range & Results \\
& & Day 1 \\
Hemoglobin, g/dL & $14.2-17.2$ & 14 \\
White blood cell count, $\mathbf{1 0}^{\wedge} \mathbf{9 / \mathbf { L }}$ & $4.2-9.4$ & 8.98 \\
Lymphocyte relative count, \% & $19.6-46.5$ & 14.6 \\
Lymphocyte absolute count, 10^9/L & $1.1-3.4$ & 1.31 \\
Neutrophil relative count, \% & $40.6-68.4$ & 82 \\
Neutrophil absolute count, 10^9/L & $2-5.8$ & 3.2 \\
Platelet count, 10^9/L & $155-320$ & 273 \\
D-dimer, ug/L & $<500$ & 1026 \\
Creatinine, mg/dL & $0.7-1.2$ & 3,1 \\
eGFR, ml/min/1,73m & & 21 \\
Sodium, mmol/L & $136-145$ & 134 \\
Potassium, mmol/L & $3.5-5.1$ & 2.35 \\
Aspartate Aminotransferase & $<40$ & 22 \\
Alanine Aminotransferase & $<40$ & 16 \\
Hs-TroponinT, ng/L & $<14$ & 89 \\
C-reactive protein, mg/dL & $<5$ & 30 \\
Il-6, pg/ml & $0-7$ &
\end{tabular}


Serum Kappa light chains, mg/L Serum Lambda light chains, $\mathrm{mg} / \mathrm{dL}$ Free light chain ratio Bence Jones proteinuria Urinary protein , $\mathrm{mg} / \mathrm{L}$ Immunofixation of urine proteins Immunofixation of serum proteins
3.3-19.4

$5.7-26.3$

$0.26-1.65$

0-150

Immunofixation of urine proteins Immunofixation of serum proteins
Immunofixation of urine protein Immunofixation of serum proteir

Author contributions: Matteo Pernigo and Marco Triggiani drafted the manuscript; Emanuele Gavazzi, Simona Fisogni, Ester Costantino and Gian Franco Pasini took part to clinical management of the case and data collection; Ilaria Papa, Alberto Vaccari, Simona Fisogni and Emanuele Gavazzi revised the manuscript.

\section{REFERENCES}

[1] Zhou F, Yu T, Du R, Fan G, Liu Y, Liu Z, et al. Clinical Course and Risk Factors for Mortality of Adult Inpatients With COVID-19 in Wuhan, China: A Retrospective Cohort Study. Lancet 2020; 395:1054-1062. doi: 10.1016/S0140-6736(20)30566-3

[2] Xiong T-Y, Redwood S, Prendergast B, Chen M. Coronaviruses and the cardiovascular system: acute and long-term implications. Eur Heart J.2020; 41:1798-1800. doi: 10.1093/eurheartj/ehaa231.

[3] Li J-W, Han T-W, Woodward M, Anderson CS, Zhou H, Chen Y-D, et al. The impact of 2019 novel coronavirus on heart injury: A systemic review and Meta-analysis. Prog Cardiovasc Dis. 2020 Apr 16; S0033-0620(20)30080-3. doi: 10.1016/j.pcad.2020.04.008.

[4] Inciardi RM, Lupi L, Zaccone G, Italia L, Raffo M, Tomasoni D, et al. Cardiac Involvement in a Patient With Coronavirus Disease 2019 (COVID-19). JAMA Cardiol. 2020; 5:1-6. doi:10.1001/jamacardio.2020.1096.

[5] Sala S, Peretto G, Gramegna M, Palmisano A, Villatore A, Vignale D, et al. Acute myocarditis presenting as a reverse Tako-Tsubo syndrome in a patient with SARS-CoV-2 respiratory infection. Eur Heart J. 2020 14; 41:1861-1862. doi: 10.1093/eurheartj/ehaa286.

[6] Kim IC, Kim JY, Kim HA, Han S. COVID-19-related myocarditis in a 21-year-old female patient. Eur Heart. 2020 14; 41:1859. doi: 10.1093/eurheartj/ehaa288.

[7] Cheng Y, Luo R, Wang K, et al. Kidney disease is associated with in-hospital death of patients with COVID-19. Kidney Int 2020; 97: 829-38. doi: 10.1016/j.kint.2020.03.005.

[8] Ronco C, Reis T, Husain-Syed F. Management of acute kidney injury in patients with COVID-19. Lancet Respir Med. 2020;S2213-2600(20)30229-0. doi: 10.1016/S2213-2600(20)30229-0.

\section{FIGURE CAPTIONS}

Figure 1: A. Electrocardiogram on admission. Sinus tachycardia with left axis deviation, slight diffuse ST depression more prominent in inferior-lateral lead.

B. Chest X-ray. Interstitial edema limited to left superior and lateral regions, and no pleural effusion.

C. Image from high resolution computed tomography scan of the lungs. Ground glass-like hyper density opacities in the peripheral portions of inferior lobes bilaterally (arrows)

Figure 2: Echocardiogram performed on day $10^{\text {th }}$

a: Parasternal long-axis view, showing moderate concentric LV hypertrophy with echo-bright appearance of myocardium. LV: left ventricle; S: septum; LA: left atrium.

b-e: 2D-speckle tracking longitudinal strain evaluation; b: bull's eye with global results. Global longitudinal strain: $-12 \%$ (18 segments). Diffuse hypokinesia is evidenced, more pronounced in basal anterior and septal 
segments (arrows), and relative apical sparing. c, d, e: longitudinal strain evaluated in apical 4-chamber, 2 -chamber, and 3-chamber views respectively, with strain curves for each segment.

Figure 3: Cardiac magnetic resonance imaging. Short tau inversion recovery (STIR) sequences in 2-chamber (A) and short-axis view (B) showed mild myocardial signal hyperintensity in left ventricle anterior wall and anterior septum in medium and apical segments (arrows), consistent with interstitial edema, confirmed and best depicted in corresponding T2-Mapping sequences (C and D, arrows).Phase sensitive inversion recovery (PSIR) sequences in short-axis (E) and 4-chamber view (F) showed diffuse intramural late gadolinium enhancement.

Figure 4: Renal biopsy specimens, PAS (Periodic Acid Schiff) stain. A: Capsular thickening and wrinkling of glomerular basement membrane (arrow). Circumferential arteriolar hyaline sclerosis (asterisk). B: Diffuse acute tubular injury with brush border loss, tubular dilatation, epithelial thinning or swollen, focal vacuolization (red arrows); partial desquamation of tubular epithelial cells (star); focal regeneration features (prominent nucleoli and mitosis - yellow arrows); modest interstitial chronic inflammation with minimal tubulitis.

Supplementary files, Figure 1: image from cranial CT scan. Several ischemic lacunar foci of the basal ganglia bilaterally (arrows), and white matter hypodensity are visible.

Supplementary files, Figure 2: Coronary angiography. Upper panels: left coronary artery. A 50\% stenosis of mid left anterior descending (LAD) artery and a 50\% stenosis of the first marginal branch of left circumflex (LCX) artery are visible. The lesions resulted non emodynamically significant (as documented by iFR - not showed). Lower panels: right coronary artery (RCA), no significant stenosis in the main vessel - posterior descending artey (PDA). Significant stenosis of a small posterior-lateral branch (PL).

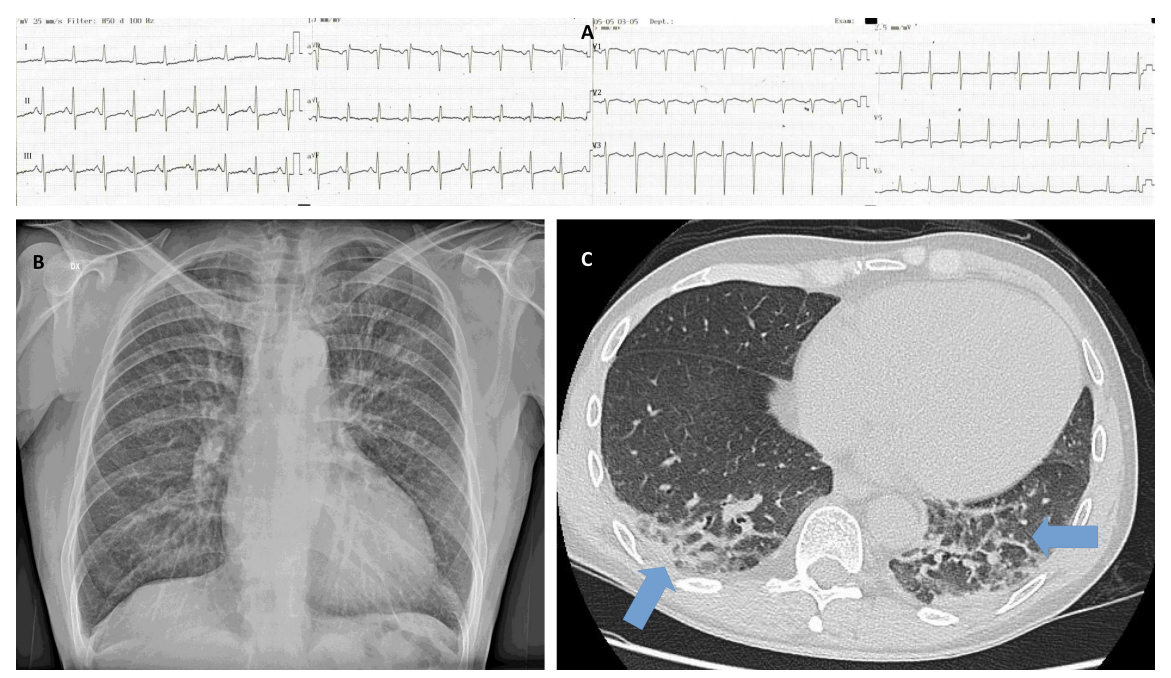




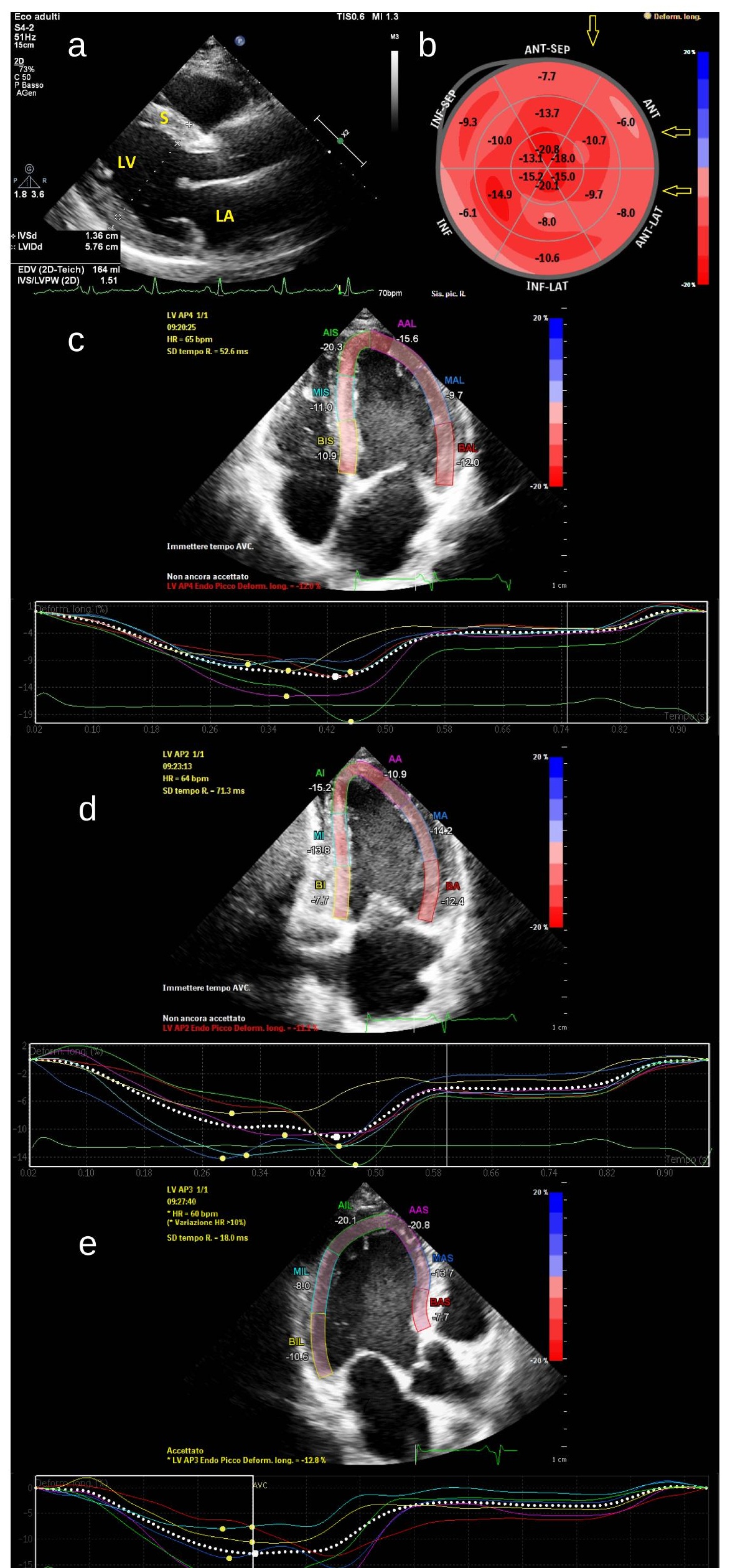




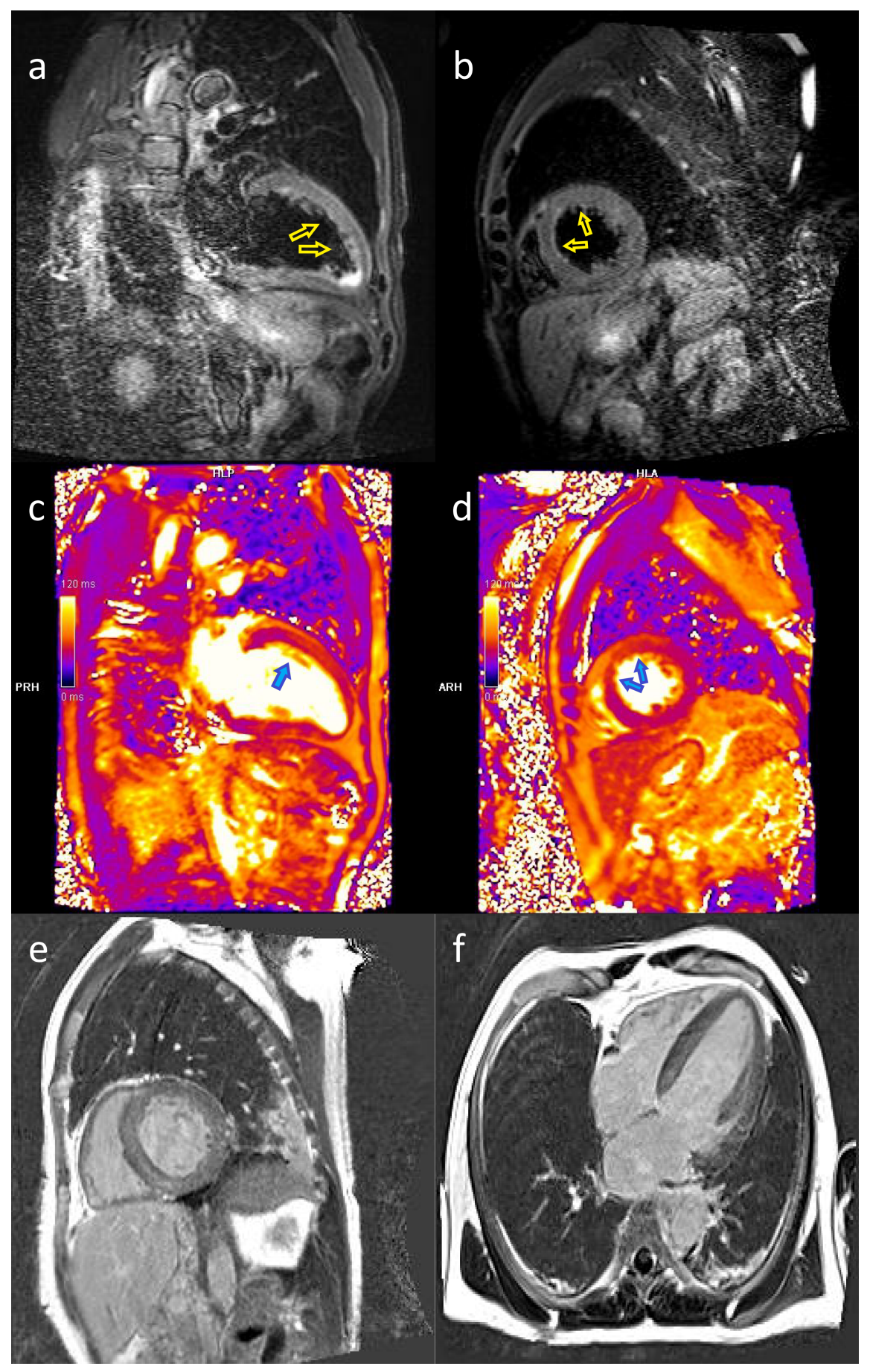




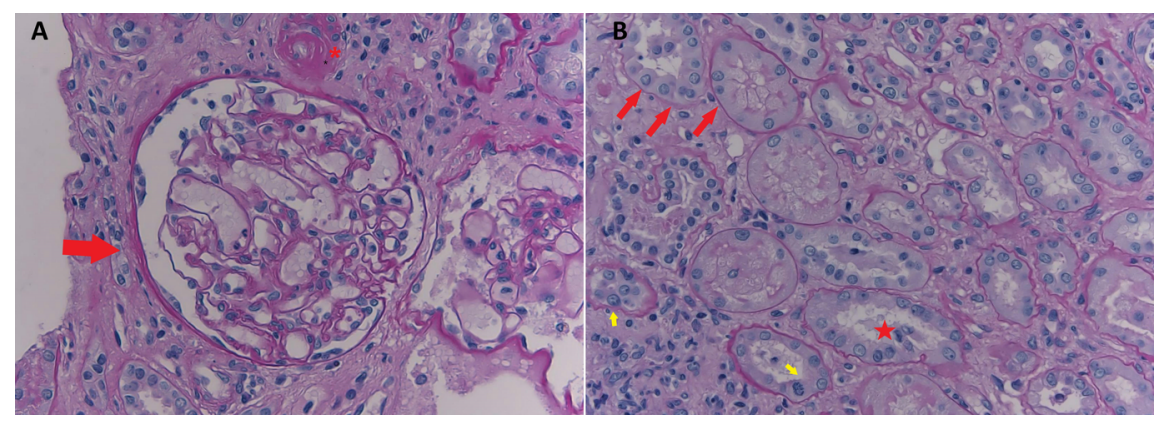

